\title{
Research of emergency cascade processes on simulation models of test and real electric power systems
}

\author{
Bulat Gaisin ${ }^{1, *}$, Ildar Shakhmaev ${ }^{2}$ \\ ${ }^{1}$ Ufa State Petroleum Technological University, Department of Electrical Engineering and Electrical Facilities of Enterprises, Ufa, Russia \\ ${ }^{2}$ Ufa state aviation technical university, Department of Electromechanics, Ufa, Russia
}

\begin{abstract}
In this work, a heterogeneous electric power system (EPS) is investigated for the possible occurrence of cascade emergency processes. In the process of computational experiments, simulation models of test and real EPS were created in which of voltages of $6,35,110,500 \mathrm{kV}$ is presented. The calculation of the trajectories of the possible occurrence and development of a cascade process in the event of a disturbance $(\mathrm{N}-1)$ in the EPS has been performed. For the investigated EPS, a model of visual representation of the heterogeneous surface of the parameters that affect the possibility of the occurrence and development of cascade processes in the EPS has been created. The study carried out on the basis of the developed methods (search for paths of the emergence and development of cascade processes, visual representation of the heterogeneous surface of EPS parameters) allows projecting the results obtained for making effective management decisions to improve survivability when designing the development of real power systems.
\end{abstract}

\section{Introduction}

At the moment, the improvement of theoretical and practical tools aimed at developing the reliability and survivability of EPS does not allow ensuring the absence of emergency disturbances of various levels of complexity, including with a massive shutdown of electricity consumers, as evidenced by the data shown in Fig. 1.

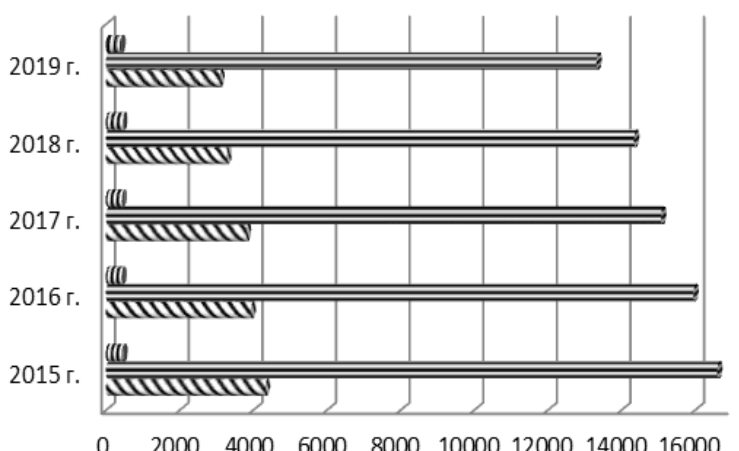

Fig. 1. Caption of the Figure 1. Below the figure.

In accordance with [1] in Fig. 1 shows statistical data indicating the regularity of accidents in the UES of Russia from 2015 to 2019 . These data indicate that, for example, in 2019 there were daily: 36.5 accidents in electrical grids of $110 \mathrm{kV}$ and above (horizontal shading); 8.44 accidents at power plants with an installed capacity of $25 \mathrm{MW}$ and above (oblique shading); 1.1 accidents that led to disconnection of consumers in the amount of $10 \mathrm{MW}$ and more (vertical shading). These statistics, according to the Ministry of Energy of Russia, show objective data on accidents in the UES of Russia, which, in turn, may not affect the reliability of electricity supply to consumers, but in fact, on average, $2-2.5 \%$ of emergency disturbances ended in disconnection of consumers of $10 \mathrm{MW}$ or more. Therefore, it is important to direct efforts to search for emergency disturbances that can weaken the survivability of the EPS and lead to a systemic accident. The emergence of other disturbances in the EPS can be admitted with the proviso that the operating personnel will do everything possible for the timely localization of the emergency disturbance and ensure that the emergency does not develop into a cascade process ending in an unacceptable mode. The proposed statistics indicate the relevance of the issue under consideration and the need to increase the requirements for ensuring the reliability and survivability of EPS.

This article proposes a tool for analyzing the possibility of the occurrence of emergency processes from the standpoint of the heterogeneity of the distribution of parameters, for specific circuit-mode conditions of the EPS. At the same time, analysis of the literature shows that in [2] the term homogeneous electrical grid means the constancy of the ratio of active resistance to inductive resistance $\mathrm{R}_{\mathrm{ij}} / \mathrm{X}_{\mathrm{ij}}$ for any grid section $(i, j, \ldots, n, i \neq j)$. But when analyzing a heterogeneous EPS, the grid parameters alone are not enough to describe the properties that affect the possibility of the occurrence and development of emergency processes.

\footnotetext{
Corresponding author: GB9688@gmail.com
} 
In the study [3], the heterogeneity was investigated using cluster, singular and spectral analysis. At the same time, EPS heterogeneities and their manifestations are considered when analyzing transient processes from the standpoint of various degrees of coherence of the motion of generators. Identification of groups of more or less coherent generators and cross-sections between them differing in weakness is considered as a method for studying heterogeneities. In this work [3], the calculations were carried out on the basis of the Jacobi matrix, which for steady-state modes depends mainly on the topology, parameters of the grid circuit, loads at the nodes and their static characteristics. It is important to note that calculations based on the Jacobi matrix cannot be effectively used for the real-time reliability assessment mode due to the need for significant computational costs.

\section{Description of terminology}

Based on the analysis of the literature [4-7], in this work, a cascade process is understood as a sequence of transitions from one quasi-stationary mode to another with step-by-step disconnection of overloaded EPS elements with termination in the range of permissible or inadmissible mode values. In case of termination in the area of unacceptable modes, the cascade process is considered part of a system (cascade) accident. The priority is to recognize and prevent the possibility of the development of a cascade process (successive shutdowns of EPS elements) into a system (cascade) accident.

According to the strict mathematical inequality proposed in [4], in the study it is assumed that a necessary condition for the existence of a cascade process is the inequality:

$$
I_{j i}+\Delta I_{j i}>I_{l i m j i}
$$

which must be performed at each subsequent step of the emergency shutdown (-s). Here $\Delta I_{j i}$ is the current surge on the overloaded element, $I_{\text {lim } j i}$ is the permissible current value for this element. Failure to fulfill inequality (1) leads to the termination of the emergency cascade process. Moreover, its termination can be both permissible and in the unacceptable range of modes.

Based on (1) and the topological arrangement of the grid elements, when calculating the EPS modes, we obtain step-by-step options for the development of cascade processes, which, in contrast to the established point of view, allows us to consider a cascade process, up to the stage of loss of controllability, as a sequence of dependent steady-state modes, objectively flowing from each other [4].

The tasks of the study led the authors to the need to formulate the following formulations characterizing the concept of EPS heterogeneity:

- EPS heterogeneity is defined as the coordinate-wise distribution of the algebraic sum of EPS parameters affecting the implementation of the cascade process over the corresponding $\mathrm{N}$-dimensional surface of the formalized system.
- The heterogeneity of the EPS depends on the circuit-mode conditions at a particular moment in time and is determined by the asymmetric and heterogeneous distribution of the set of parameters characterizing the EPS elements according to the EPS circuit.

- The heterogeneity of the EPS determines the possibility of the occurrence and development of emergency cascade processes and depends on the magnitude and topological distribution of the EPS parameters according to the system diagram.

At first glance, it may seem that the concept of heterogeneity proposed above was formulated vaguely, but in reality this formulation offers a very specific one, incl. mathematical and 3D-graphic tools for the analysis of EPS heterogeneity [8, 9].

\section{Creation of simulation models of test and real EPS}

Based on the proposed concepts of the cascade process and the concepts of heterogeneity, simulation models of test and real EPS were created. In this work, the test scheme of an EPS (N nodes) is understood as a scheme that, as a result of a number of mathematical studies, reveals the regularities of the influence of the arrangement of elements according to the EPS topology on the possibility of cascade processes. The mathematical apparatus used to create simulation models is described in detail in $[8,9]$, so the reader can refer to the above links for details.

The EPS model for studying heterogeneity, as well as the occurrence and development of cascade processes can be divided according to the voltage scale. The first model (Fig. 2) has 25 nodes and $U_{\text {nom }}=110 \mathrm{kV}$, the second model (Fig. 3) characterizes approaches to backbone grids and has 36 nodes, 60 branches and $U_{\text {nom }}$ $=500 \mathrm{kV}$. The third model (Fig. 4), characterizes distribution grids with elements of distributed generation present in them, this model has 40 nodes and 67 branches $U_{\text {nom }}=10 \mathrm{kV}, 35 \mathrm{kV}, 110 \mathrm{kV}$. The fourth model (Fig. 5) was created according to the developed methods [8-13] on the basis of a real EPS and describes the sequence of disconnection processes of grid elements during the development of a cascade, systemic accident that occurred on 2.07.2016 in the Republic of Bashkortostan (RB).

In the diagram shown in Fig. 2 (a) the scheme simulates a cascade process consisting of 7 steps by making changes to the distribution of parameters according to the scheme of an heterogeneous EPS. In fig. 2 (b) the value of the maximum permissible current $I_{p . c}$. for the EPS element is represented by histograms with oblique shading. Histograms with vertical shading indicate the value of the current $I_{\text {initial conditions for the }}$ corresponding element before the disturbance that led to the cascade failure in the grid. The horizontal shading represents the histograms indicating the $I_{\text {acting }}+\Delta I$ values for the EPS elements. In this test model of the 


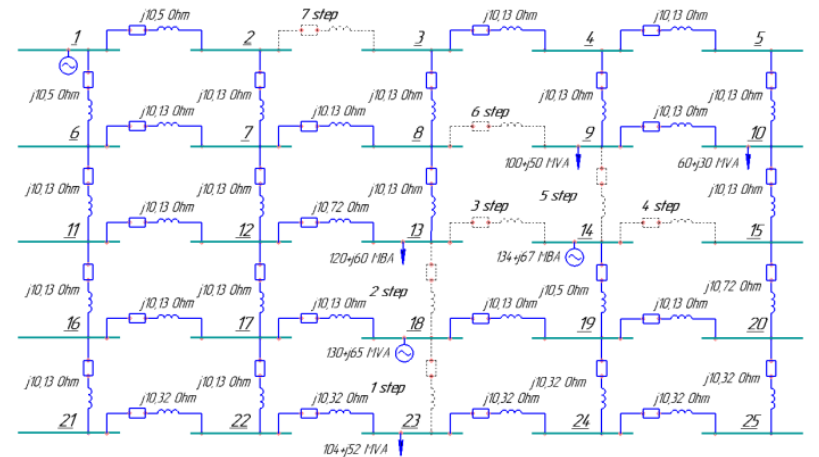

a)

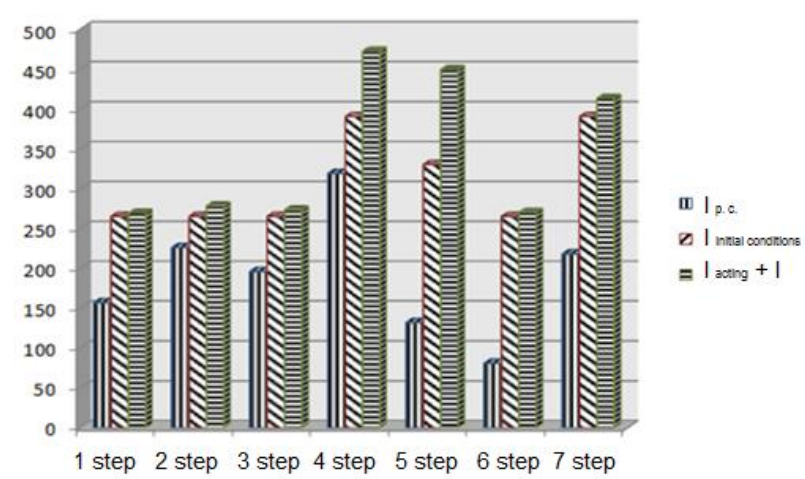

b)

Fig. 2. Test twenty-five nodal EPS circuit $\left(U_{\text {nom }}=110 \mathrm{kV}\right)$, for studies of the influence of EPS heterogeneity on the possibility the occurrence and development of emergency cascade processes. Figure: 4 - Test 40-nodal EPS scheme with RG elements.

EPS, at time $t_{l}$, a trigger disturbance occurred due to an increase in power consumption in node 23 from $52+j 26$ $M V A$ to $72+j 36 M V A$, which led to current overload of branch 18-23. In this case, the parameters of the branch 18-23 are represented by the brand of the AC-70/11 wire with the parameters: $R=10.7 \mathrm{Ohm}, X=10.13 \mathrm{Ohm}$, $B-255 \cdot 10^{-6} \mathrm{~S}$. In the branch $18-23$, the ratio of the flowing current to the maximum allowable current is 1.02 , which indicates that the maximum allowable value has been exceeded and the overloaded element is disconnected in accordance with condition (1). This study is presented in more detail in [8].

In fig. 3 shows a test 36-nodal diagram of an integrated EPS (IES) in which the conditions for the flow of active power of $2868 \mathrm{MW}$ between EPS 1 (left 3 columns of the grid diagram nodes) and EPS 2 (right 3 columns of the grid circuit nodes) are modeled. On this simulation model, studies were carried out to increase survivability by changing the power flows between power plants with cheap and expensive electricity generation costs, due to which the current unloading of branches close to the limiting values was carried out.

When calculating the cost of raising expensive generation, we use the following data [14]:

- electricity is considered cheap if it is produced at the rate of 310 grams of standard fuel per $1 \mathrm{kWh}$, we take its cost of 3.9 rubles for $1 \mathrm{~kW} \cdot h$;

- electricity is considered expensive if it is produced at the rate of 650 grams of standard fuel per $1 \mathrm{~kW} \cdot h$, we take its cost as 5 rubles per $1 \mathrm{~kW} \cdot h$.

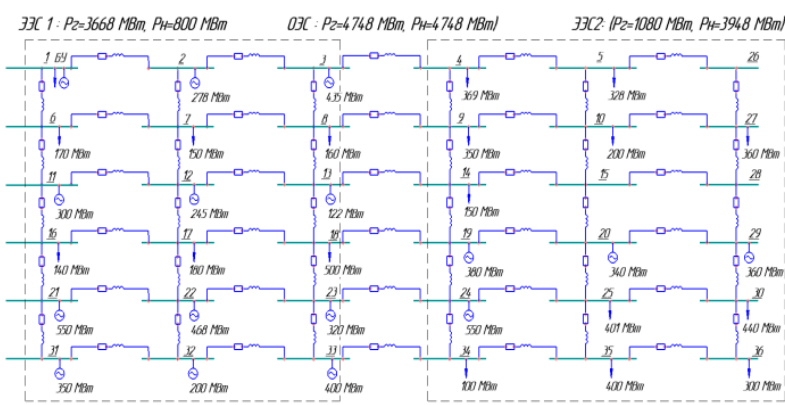

Fig. 3. Test thirty-six nodal diagram of the backbone grid oa the united EPS $\left(U_{\text {nom }}=500 \mathrm{kV}\right)$.

Also, using the example of this (Fig. 3) simulation model, a study was carried out to improve the survivability of the UPS by using devices related to flexible AC power transmission (FACTS), taking into account a 2-fold decrease in the reactance of one intersystem branch and a corresponding increase in the power flow between EPS 1 and EPS 2. Further calculations of a feasibility study were performed in accordance with [15], as a result of which a comparative justification of the cost indicators of FACTS devices, a standard solution for the construction of a new $500 \mathrm{kV}$ line, and the possibility of changing flows by redistributing the load of generators at power plants with expensive and cheap generation electricity.

At the next stage of research, a 40 nodal EPS scheme was created with $U_{\text {nom }}=10 \mathrm{kV}, 35 \mathrm{kV}, 110 \mathrm{kV}$ (Fig. 4), which includes distribution grids (DGr), as well as elements of distributed generation (DGen), as a factor of a possible increase vitality.

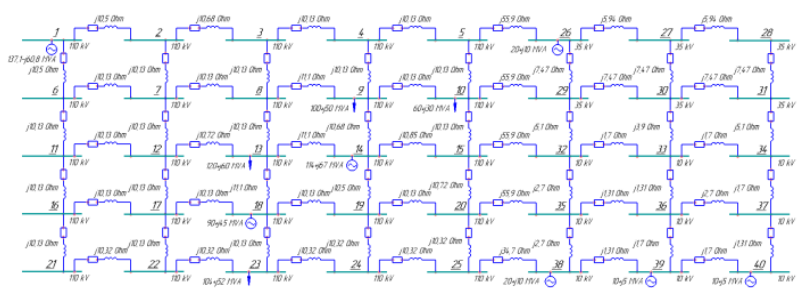

Fig. 4. Test forty nodal scheme of EPS with DG elements $\left(U_{\text {nom }}=10 \mathrm{kV}, 35 \mathrm{kV}, 110 \mathrm{kV}\right)$.

In this model (Fig. 4), on the basis of studies (Fig. 2), a similar cascade process is modeled, consisting of emergency shutdowns of 7 elements of the EPS in accordance with condition (1). On the basis of this model, the cascade process was prevented by connecting power plants based on RG elements (Node (N) 38 $20 \mathrm{MW}, \mathrm{N} 39$ - 10 MW, N 40 - 10 MW, N 26 - 20 MW). This study shows the applicability of the proposed tools to DGr with elements of the DGen.

The task of the next simulation model (Fig. 5) was to demonstrate the application of the above proposed mathematical models and test circuits based on a real EPS, in which a cascade system accident occurred on July 2, 2020. In fig. 5 shows a 3D-graphic model of the surface of the heterogeneous distribution of the current parameter (I, A) in the elements of the EPS RB during the step-by-step development of a cascade accident, after disconnecting the $500 \mathrm{kV}$ Buiskaya - Ufimskaya overhead line for $110 \mathrm{kV}, 220 \mathrm{kV}, 500 \mathrm{kV}$ grid elements. 


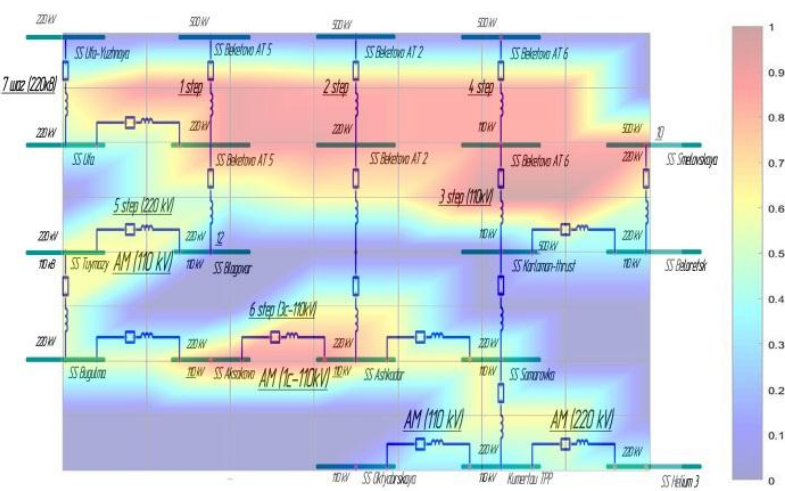

Fig. 5. An equivalent circuit (without fictitious nodes and branches) and a 3D-graphic surface of the heterogeneous distribution of the current parameter I (A) of the investigated power region at the moment of disconnection of the $500 \mathrm{kV}$ Buiskaya-Ufimskaya power transmission line, for grid elements of $110 \mathrm{kV}, 220 \mathrm{kV}, 500 \mathrm{kV}$.

In fig. 5 shows an heterogeneous surface in terms of the current parameter (I, p.u.), which flowed in the EPS elements during the step-by-step development of the cascade process. The nodes indicate $U_{\text {nom }}=110 \mathrm{kV}$, $220 \mathrm{kV}, 500 \mathrm{kV}$ of the disconnected elements, some of the branches of the EPS RB are replaced with equivalents corresponding to the real scheme, the nodes indicate the connections with consumers and power plants, in parentheses the node belongs to the neighboring EPS (Tatarstan - TE, Chelyabinsk - ChE, Orenburg - OE). After the 7th step of disconnection of overcurrent elements, an asynchronous mode (in Fig. 5, the AM is indicated) started on 4 links $110 \mathrm{kV}, 220 \mathrm{kV}$ between the EPS of the RB and the UES of Russia, as a result of which the automatic elimination of the asynchronous mode (AEAM) was triggered to turn off.

In accordance with [16], as a result of a cascade accident, the Central part of the EPS RB separated into operation isolated from the UES of Russia with a power deficit of $866 \mathrm{MW}$ and a decrease in frequency at the time of separation to $47.47 \mathrm{~Hz}$, while the automatic frequency unloading (AFU) shut off consumers in the amount of $967 \mathrm{MW}$.

As a result of cascade outages, there was a decrease in the load of consumers in the EPS: RB (710 MW), Chelyabinsk (280 MW) and Orenburg (5 MW) regions, which in total amounted to $995 \mathrm{MW}$. The decrease in the load of consumers occurred not only as a result of the action of emergency control automation, but also due to the action of technological protections.

During the operation of the AFU at the facilities of the EPS RB, cases of malfunctioning (failure to operate on disconnection) were recorded for a total of $102 \mathrm{AFU}$ devices (434 connections), with a total load of connected consumers in the amount of $194 \mathrm{MW}$, accounting for $22 \%$ of the total number of connections connected under the action AFU, the settings of which worked during the development of the accident. Failure of AFU devices with higher frequency settings led to the operation of AFU devices with lower settings and disconnection of additional consumers.

The described model of the development of a cascade accident (Fig. 5) indicates the efficiency and applicability of the developed methods to real EPS schemes. At the same time, this model shows the analysis of a step-by-step transition from one quasisteady-state regime to another according to condition (1), until the moment when asynchronous regimes appear.

\section{Conclusion}

The paper presents simulation models of test and real EPS on the basis of which studies were carried out in accordance with the methods and techniques developed in $[4,8]$ for determining the influence of EPS heterogeneity on the occurrence and development of emergency cascade processes, which make it possible in practice to determine the possible course the development of a cascade accident and ensure the survivability (reliability) and efficiency of the operation of power nodes and electrical grids, as well as increase the efficiency of decisions taken in the operational control of modes and design of EPS.

\section{References}

1. Statistics on emergency outages in the power system of the Russian Federation // Ministry of Energy of the Russian Federation [Electronic resource]. URL: https://minenergo.gov.ru/node/267 (date accessed: 09.10.2020).

2. S. Ananicheva, A. Myzin, Methods of analysis and calculation of closed electrical grids. 4th ed., Rev. Ekaterinburg: UrFU, 94 (2012).

3. O. Voitov, N. Voropay, A. Gamm, I. Golub, D. Efimov, Analysis of heterogeneities of electric power systems. Novosibirsk: Science. Siberian Publishing Company RAS, 256 (1999).

4. I. Shakhmaev, On the ways to prevent cascade processes in power systems. Vestnik USATU, 13, No. 1, 176-179 (2009).

5. Reliability and survivability of the power system // System operator of a single power system [Electronic resource]. URL: https://www.soups.ru/index.php?id=rza_goals_rel (date accessed: 09.10.2020).

6. Y. Rudenko, V. Semenov, Automation of dispatch control in the power industry. M .: MEI, 648 (2000).

7. A. Burman, Y. Rozanov, Y. Shakaryan, Management of electricity flows and improving the efficiency of electric power systems. Moscow: MEI, 336 (2012).

8. B. Gaisin, Development of methods for determining the influence of heterogeneity of electric power systems on the emergence and development of emergency cascade processes: Ph.D. thesis. Moscow, 156 (2019).

9. I. Shakhmaev, B. Gaisin, N. Novikov, Methods for detecting cascade processes in electric power systems // 91st meeting of the seminar on the topic "Methodological and practical problems of reliability of power systems", 1-10 (2019). 
10. B. Gaisin, A method of making effective decisions to increase survivability in the design and development of power systems // Vestnik USATU, Electrical Engineering, 21, No. 3 (77), 47-53 (2017).

11. I. Shahmaev, B. Gaisin, O. Shiryaev. A new method of taking management decisions at designing and developing electric power systems // 2nd International Conference on Industrial Engineering, Applications and Manufacturing (ICIEAM), 1-7, (2016).

12. F. Ismagilov, I. Shahmaev, B. Gaisin, The technique to improve the effectiveness of control systems in inhomogeneous electric power grids // International Siberian Conference on Control and Communications (SIBCON), 1-7 (2017).

13. I. Shakhmaev, B. Gaisin, Methods for identifying (recognizing) emergency cascade processes in electric power systems // Vestnik USATU, Electrical Engineering, 22, No. 2 (80), 97-104, (2018).

14. Cost indicators of electricity generation // Open Joint Stock Company "Administrator of the Trading System of the Wholesale Electricity Market" [Electronic resource]. URL: http://www.atsenergo.ru (date of access: 09.10.2020).

15. Methodology for assessing the technical and economic efficiency of using FACTS devices in the UNEG of Russia. Moscow, 35 (2009).

16. Act No. 01 of investigation of the causes of the accident that occurred on 02.07.2016, Ufa, 88 (2016). 